\title{
Pour une anthropologie symétrique entre " développeurs » et " développés »
}

"Developees" and "Developers": for a Symmetric Anthropology

\section{Philippe Lavigne Delville}

\section{OpenEdition}

12 Journals

Édition électronique

URL : http://journals.openedition.org/etudesafricaines/16752

DOI : 10.4000/etudesafricaines. 16752

ISSN : $1777-5353$

Éditeur

Éditions de l'EHESS

\section{Édition imprimée}

Date de publication : 8 septembre 2011

Pagination : 491-509

ISBN : 978-2-7132-2298-6

ISSN : 0008-0055

Référence électronique

Philippe Lavigne Delville, «Pour une anthropologie symétrique entre « développeurs » et "développés » », Cahiers d'études africaines [En ligne], 202-203 | 2011, mis en ligne le 27 octobre 2013, consulté le 20 juin 2020. URL : http://journals.openedition.org/etudesafricaines/16752 ; DOI : https:// doi.org/10.4000/etudesafricaines.16752

Ce document a été généré automatiquement le 20 juin 2020.

(c) Cahiers d'Études africaines 


\title{
Pour une anthropologie symétrique entre « développeurs » et « développés »
}

\author{
"Developees" and "Developers": for a Symmetric Anthropology
}

\author{
Philippe Lavigne Delville
}

1 Pour de nombreux anthropologues, l'échec des projets de développement semble programmé. Selon les auteurs, il découle quasi mécaniquement du manque de connaissance des acteurs locaux, de finalités trop décalées par rapport aux réalités locales, quand ce n'est pas du poids des acteurs dominants (bailleurs, État, pouvoirs locaux selon les cas) ou de la perversité structurelle du système d'aide et de son projet hégémonique. Selon une telle conception, les dispositifs d'aide, les agents de développement, leurs stratégies d'action, les pratiques effectives n'ont guère d'autonomie et finalement guère d'intérêt. De fait, autant les analyses autour des projets de développement ou " du développement » sont nombreuses, autant les études anthropologiques de dispositifs d'intervention sont rares, en particulier en français.

2 À partir de l'analyse de deux études anthropologiques sur des interventions d'oNG ${ }^{1}$, je voudrais montrer ici que l'absence d'intérêt porté aux dispositifs d'intervention et aux développeurs comme objets de recherche pose des problèmes dès lors que les anthropologues affirment analyser ces interventions et leurs effets, et ce d'autant plus qu'elle se combine avec un populisme plus «idéologique " que "méthodologique $»^{2}$ : des problèmes méthodologiques voire déontologiques, au sens où cela induit des lectures partielles, parfois biaisées ou hautement discutables des projets étudiés ; des problèmes scientifiques au sens où cela laisse dans l'ombre des dimensions importantes de ce qu'est l'intervention de développement en pratique ${ }^{3}$. 


\section{La méconnaissance, par les développeurs, des logiques sociales locales suffit-elle à démontrer l'existence d'effets pervers?}

3 Se demandant avec ironie si l'on n'a pas là « des paysans plus professionnels que les développeurs?», Claude Arditi (2004: 841) constate que «développeurs d'hier et oNG actuelles, qui "conseillent" les paysans afin qu'ils deviennent plus professionnels ont en commun une absence totale de désir de connaissance des sociétés locales ». Dans une brillante synthèse d'une dizaine de pages sur les stratégies paysannes des paysans sara $\mathrm{du}$ sud du Tchad, il montre que la finalité sociale de la production n'est pas l'accumulation de richesse, du fait d'un «ethos de la consommation immédiate intimement lié à une exigence de redistribution à laquelle sont soumis les riches" (ibid.: 844). Ce qui lui permet de critiquer, en trois pages chacune, les postulats et les modes d'action de deux ONG, l'AFDI (Agriculteurs français et développement international, ONG d'agriculteurs français) ${ }^{4}$, et le BELACD, ONG tchadienne d'épargnecrédit.

\section{Les représentations des développeurs ont-elles un impact sur les stratégies locales d'appropriation de l'offre de l'ong ?}

ONG tchadienne d'épargne-crédit, le BELACD porte une vision de paysans entrepreneurs, ayant besoin de crédit pour produire plus, accumuler et investir. Or, les paysans sara sont dans des logiques sociales de destruction du surplus et non de capitalisation : « On peut s'étonner de ce que cette faible capacité d'épargne, connue depuis longtemps, n'ait pas suscité davantage de réflexions de la part de ceux qui mettent en œuvre ces programmes, comme si les représentations et les comportements vis-à-vis de l'argent étaient universels et n'étaient affectés ni par la culture ni par l'histoire " (ibid.: 861-862). Cette méconnaissance témoigne pour Arditi d'un manque de professionnalisme qui génère des effets pervers.

5 L'argument semble incontestable. Il pose cependant des questions de deux ordres. La première porte sur la notion de professionnalisme. Arditi considère que la connaissance anthropologique en est un critère-clé. On s'accordera sur le fait que cela devrait en faire partie. Mais le professionnalisme se définit avant tout en référence aux cadres conceptuels et méthodologiques reconnus au sein d'un secteur professionnel donné et à leur inégale maîtrise par ses membres, à un moment donné de son histoire. Le jeune secteur de la microfinance (à peine 20 ans) a connu un important travail de définition d'outils et de "bonnes pratiques", sous l'égide des institutions internationales. À partir d'un raisonnement simple (sinon simpliste) sur l'offre de crédit et ses effets supposés, la question des décalages entre usages affirmés et usages réels du crédit s'est imposée progressivement et a été intégrée aux outils de suivi de clientèle au début des années $2000^{5}$. Dès lors, s'il ne l'a pas fait en 2003, le BELACD est sans doute légèrement en retard, mais pas encore en décalage massif par rapport aux critères de professionnalisme de la microfinance, même si on peut effectivement penser qu'un souci plus affirmé de comprendre les logiques de ses clients aurait pu l'amener à le découvrir par lui-même. 
6 Si l'adéquation des produits offerts à la clientèle et les enquêtes de satisfaction font aujourd'hui normalement partie des références professionnelles du secteur, ce n'est effectivement pas le cas pour l'analyse socio-anthropologique des finalités sociales de la richesse chez les clients. Mais une telle impasse a-t-elle réellement des incidences négatives fortes sur l'adéquation de l'offre? C'est mon second point, celui du rapport entre représentations, pratiques et effets des pratiques. Arditi (ibid.: 861) a parfaitement le droit d'être choqué par le fait que les agents du BELACD jugent " archaïques » les comportements de consommation et de destruction de surplus des paysans, je le serais aussi ${ }^{6}$. Mais si le sujet de son article est bien « la pertinence et de l'efficacité des diverses actions de développement mises en œuvre par des ONG » (ibid.: 842), et dès lors qu'un certain décalage entre les discours institutionnels justifiant l'intervention et les réalités locales existe toujours, la question principale n'est-elle pas celle des pratiques effectives et de ce qu'elles produisent? La question des représentations n'ayant finalement d'intérêt par rapport à la question de l'efficacité que dans la mesure où ces représentations structurent des actions à effet pervers avéré : après tout, les clients du BELACD se moquent sans doute bien des justifications théoriques que cette ONG se donne et ils ont bien raison.

7 Si les crédits sont destinés aux femmes, qui ne sont pas (ou pas de la même façon) dans ces logiques de destruction de surplus, et qu'ils leur permettent de réguler leur trésorerie et de développer leur activité économique et l'atteinte autonome de leurs objectifs sociaux ; si les crédits de campagne permettent aux petits paysans de sécuriser leur production et leur revenu, et d'avoir plus de surplus à détruire et de bière à boire, satisfaisant ainsi mieux leurs logiques sociales, le fait que l'ong reste dans une illusion en imaginant un usage productif du crédit est-il problématique ? Bref, au-delà du fait qu'il choque l'anthropologue, ce décalage entre discours professionnel et réalités est-il un réel problème pour les paysans sara, et si oui, en quoi ? Après tout, ne favorise-t-il pas la liberté des paysans sara d'affecter le crédit à quoi bon leur semble (dans la bière, dans la trésorerie, dans l'entretien des réseaux sociaux, en plus de l'engrais et des charrues) dans un malentendu finalement productif de leur point de vue?

Affirmer que les paysans sara ne sont pas, contrairement aux souhaits du BELACD, dans une logique d'accumulation et de maximisation du profit ne suffit donc pas pour démontrer qu'une offre d'épargne et de crédit n'a pas d'utilité pour eux ou induit nécessairement des effets pervers. Il faudrait pour cela analyser la diversité des clients, leurs pratiques, la façon dont ils intègrent cette offre nouvelle dans leurs stratégies d'épargne et de recours au crédit (à côté des prêts familiaux, des emprunts de solidarité, des crédits de campagne, des éventuels recours à l'usure, etc.). Et pour cela entrer dans l'analyse des produits offerts (montants, dates, modes de remboursements, taux d'intérêt, etc.), des règles du jeu proposées par l'intervenant (modalités d'octroi du crédit, système de caution, modalités de remboursement, etc.), des pratiques effectives des agents de crédit (qui peuvent être significativement différentes de la norme, avec des conséquences positives ou négatives pour les clients), et finalement des stratégies des clients par rapport à cette offre. Or, si Arditi reproche au BELACD de ne pas analyser les effets réels de son intervention, il ne le fait pas davantage, se contentant d'un discours général, a priori, qui fait lui aussi fi des différenciations sociales à travers les usages différenciés de l'épargne et du crédit. On ne saura donc pas si l'offre de crédit est utile ou non à certains paysans sara, à quoi, à quel prix pour eux. 
On ne saura pas non plus si les représentations des agents de cette ONG ont une influence quelconque sur cette utilité.

\section{Mais au fait, qui propose le crédit?}

9 L'absence des acteurs et des dispositifs institutionnels dans l'analyse est plus frappante encore : à lire Arditi, les agents du BELACD semblent directement impliqués dans la distribution et la gestion du crédit. Pourtant, la seule source bibliographique qu'il cite sur ce cas (Huchon 2000) explique très clairement que, si le BELACD a bien été à l'origine de l'offre de crédit, celle-ci est actuellement gérée par des Clubs d'épargne et de crédit, sous le chapeau d'une Union des clubs d'épargne et de crédit (UCEC) (ibid. : 24), faisant de celle-ci une «banque paysanne sur principes coopératifs» (ibid.:27) pilotée par des bénévoles paysans et un seul salarié, le BELACD n'ayant plus qu'un rôle d'appui. Ce n'est pas du tout la même chose! En effet, sauf à démontrer que cette UCEC n'est qu'une interface neutre, reprenant sans réinterprétation les mots d'ordre du BELACD, ce sont bien les règles du jeu de l'UCEC, la stratégie de ses leaders paysans, et les pratiques des responsables des « clubs » locaux qui déterminent l'offre de crédit et ses modalités. Dès lors, la méconnaissance des paysans par les agents du BELACD ne peut avoir d'impact concret que si elle est intériorisée par les responsables paysans de l'ucEc eux-mêmes... ce qui serait surprenant ou en tous cas à expliquer: invoquer les préjugés des techniciens vis-à-vis des paysans ne suffit clairement plus.

\section{Pourquoi l'échec : produit de l'histoire ou résultat nécessaire d'une conception « technico- gestionnaire »?}

10 À travers l'analyse de l'échec d'un projet d'électrification rurale, Alizés Électrique, Sophie Caratini (2005) veut mettre en avant la réalité du « rapport de développement ", c'est-à-dire (bien qu'elle ne le définisse jamais explicitement) le rapport politique d'ingérence et de domination inhérent à la relation « d'aide ».

Alizés Électrique a été conçu et mis en œuvre par une ong française, le Gret, entre 1995 et 1997 dans le Trarza mauritanien sur financement PNUD ${ }^{7}$. Dans la suite d'une action sur le pompage éolien et sur le même schéma, son objectif était de mettre en place dans la même région un service de l'électricité. Un petit réseau ou un système de recharge de batteries alimenté par des aérogénérateurs devait permettre aux ménages abonnés d'alimenter quelques ampoules et une télévision ou un réfrigérateur, un comité de gestion issu de l'assemblée villageoise (la jemaa) assurant la gestion du service.

\section{Alizés Électrique, un projet illustratif d'une logique « technico- gestionnaire »?}

En 2002, lors des enquêtes de l'auteure ${ }^{8}$, l'échec est patent. Ce projet et son échec semblent à l'auteure illustratif d'une « logique de kit technico-gestionnaire » (ibid.: 91) : "Pour "réussir" - ou tenter de prévenir l'échec -, les schémas techniques sont doublés de schémas de transformation de la société. [...] Le financement du progrès est désormais conditionné par la soumission des populations à des modèles d'organisation 
issus d'une pensée technico-gestionnaire unique qui met les individus "sous contrat" et distribue les droits et devoirs de chacun, y compris celui de la sanction » (ibid.: 75). La tentative de concrétiser ce "modèle d'organisation sociale» (ibid.: 91) "a créé des désordres, et par endroits des conflits difficilement réparables. [...] Les "comités de gestion" doublent les organisations sociales et plus encore politiques existantes sans tenir compte des clivages qui les traversent, et créent un second lieu décisionnel, qui ne recoupe pas toujours exactement le premier qui est généralement plus étroit et plus hiérarchisé. [...] Des jalousies ont été suscitées, des froissements ont été ressentis, susceptibles de provoquer des oppositions sourdes et d'affecter les relations villageoises en général. Des discussions à n'en plus finir ont opposé les familles, des femmes se sont heurtées, des enfants se sont bagarrés » (ibid. : 80-81).

\section{Biais structurels ou produit de l'histoire?} « technico-gestionnaire » couplée à une démocratisation imposée dans la composition des comités, et même plus fondamentalement de ces rapports de domination liés à l'aide et de la résistance parallèle des villageois et de l'état. Dès lors, pas besoin de s'encombrer des détails de l'histoire.

14

(t) 'equipe-projet avait déjà une de la région et de ses acteurs, à travers un projet antérieur, centré sur l'eau potable, reconnu à l'époque comme un succès. Elle a fait appel aux débuts à un chercheur sociologue mauritanien, pour réfléchir à sa démarche, a tenté de définir les règles du jeu avec la jemaa des villages. Fin 1997, à la fin de la première phase de deux ans, différentes études de terrain donnaient une image positive de l'action. Le dispositif local d'électrification fonctionnait correctement, les clients, que personne n'obligeait à s'abonner, goûtaient « aux bienfaits de l'éclairage électrique » (ibid.: 81), les gérants s'investissaient dans leur tâche. Les équipements et les modes de gestion n'étaient pas tout à fait au point, ayant demandé plus de temps que prévu à expérimenter et stabiliser (potentiel éolien moins fort que prévu, problèmes techniques variés, fiabilité de batteries, etc.). La stabilisation de l'ensemble demandait de mettre en place le dispositif de maintenance, ce qui était prévu pour la phase suivante. La continuité de l'appui devait permettre de travailler les problèmes rencontrés par les acteurs locaux, tant sur les batteries (fiabilité, transport) (ibid. : 80 ; Matthieussent et al. $2005: 77$ ) que sur les choix organisationnels au niveau des comités de gestion, et de consolider le dispositif institutionnel. Cette seconde phase, que l'AFD affirmait vouloir financer, devait aussi permettre, une fois le dispositif technique stabilisé, de faire entrer dans le jeu Nassim, la coopérative régionale qui assurait avec efficacité la maintenance des éoliennes de pompages, et qui voyait là une opportunité d'élargir son volume d'activités, stabilisant ainsi le dispositif global.

Fin 1997, à l'issue de ces deux ans, il ne semblait pas y avoir de signes évidents d'un échec en germe. Les conflits politiques locaux et les ambiguïtés des comités de gestion n'apparaissaient pas rédhibitoires, ni à l'équipe ni aux observateurs externes. Le degré d'autonomie des dispositifs, après deux ans d'expérimentation, n'avait rien d'inquiétant. Il y avait une stratégie d'autonomisation, sur un pas de temps de 6 ou 8 ans, réaliste pour une telle innovation, que la reprise du financement par l'AFD devait rendre possible. 
16 Évidemment, rien ne garantit que ce scénario aurait «marché ». Mais le fait est qu'il existait et n'a pas pu être tenté. En effet, la continuité de l'action a été rendue impossible par une série de retournements institutionnels. L'AFD, qui voulait financer la suite du projet qu'elle avait évalué très positivement, a suscité la création d'une «cellule de l'électrification décentralisée » (CELED) au sein du ministère de l'Énergie, pour renforcer la maîtrise d'ouvrage publique sur ce projet. Les blocages de la CELED l'ont ensuite amenée à demander la création d'une Agence pour le développement de l'électrification rurale (ADER) censée être plus autonome et plus efficace. Pendant ce temps, l'AFD a certes financé un suivi léger du Gret, mais sans moyens pour poursuivre le travail de terrain. L'équipe a observé sans pouvoir agir la dégradation progressive d'un service non encore stabilisé, le non-renouvellement des batteries par une CELED non fonctionnelle, la chute progressive des abonnements. L'étude commandée par le Gret en 2001 (Rocher 2002, résumée dans Matthieussent et al. 2005 : 75-81) montre une satisfaction initiale par rapport à l'électricité, et la déception des villageois devant la dégradation du service, ainsi que leurs tentatives pour y faire face: lorsque la maintenance n'a plus été assurée, les villageois ont continué à payer leur abonnement pendant un temps, puis ont cessé devant la baisse de durée de vie des batteries, non compensée par leur remplacement ; certains ont acheté des kits solaires (Matthieussent et al. $2005:$ 79, 81, 92). Caratini (2005: 81) reconnaît elle-même ce "rapport de confiance longtemps maintenu entre "le projet" et les villageois", l'amertume des "villageois ayant quelque temps goûté aux bienfaits de l'électricité » (ibid.). Autant de signes qui cadrent mal avec l'hypothèse d'un refus radical. Plus qu'un rejet de la part d'acteurs locaux enfin libérés de l'ingérence du Gret et de l'obligation de s'y soumettre en façade, et qui auraient auparavant été incapables d'exprimer leur rejet, y compris aux missions externes, l'image qui ressort de cette période de 1998 et 1999 est celle d'une déliquescence progressive du dispositif au fur et à mesure des pannes, déliquescence inévitable dès lors que des maillons essentiels de sa fonctionnalité (la stabilisation technico-organisationnelle, d'une part, la maintenance d'autre part, qui impliquait des institutions externes) n'existaient pas encore et que les transitions sans financement entre le projet pilote, la CELED puis l'ADER n'ont pas permis d'y répondre.

17 Dès lors, qu'est-ce qui explique l'échec? Les biais structurels ou l'histoire et les aléas institutionnels, qui ont empêché d'aller au bout de sa logique et de travailler à stabiliser un dispositif institutionnel dont il avait posé les bases? Il y a certainement eu des tensions, contradictions et conflits, larvés ou non, suscités par les comités de gestion, même si on peut penser qu'ils ont été aggravés par la déliquescence du service ${ }^{9}$. Il existe de réelles ambiguïtés sur le modèle "communautaire ", largement prévalent à l'époque dans le milieu du développement. Nombreux sont les projets qui nient les impasses structurelles de leurs choix et invoquent un peu facilement les conditions externes pour expliquer l'échec. Pour autant, il semble assez évident (et les rapports d'activités du projet, témoignant des difficultés non prévues et du travail pour y remédier, le montrent bien) que deux ans ne suffisent pas à expérimenter et à stabiliser un système d'électrification rurale, nouveau dans le contexte mauritanien de l'époque, et que l'interruption de l'appui n'a pas permis d'arriver au stade de l'épreuve de vérité.

18 Dès lors ne doit-on pas, d'un point de vue de rigueur interprétative, s'interroger sur la hiérarchie des causes dans un processus complexe : à partir du moment où l'histoire du processus permet d'expliquer, pour partie au moins, les faits observés en 2002, est-il 
rigoureux de faire l'impasse dessus pour privilégier a priori une hypothèse structurelle? Autrement dit, la thèse de Caratini n'aurait-elle pas été plus convaincante si elle avait pris en compte cette histoire et ce qu'elle explique, au moins en partie, et tenter dans ses enquêtes locales de distinguer les éventuelles évolutions des perceptions selon les périodes?

Une telle mise en perspective aurait été d'autant plus utile que l'organisation locale de gestion des générateurs (et plus généralement le dispositif couplant comité de gestion villageois et coopérative de maintenance) n'a pas été inventée dans un bureau par des ingénieurs raisonnant de façon abstraite des systèmes de gestion. Ces dispositifs sont issus d'une expérience antérieure de mise en place d'éoliennes de pompage, dans la même région du Trarza, projet qui avait abouti à des résultats jugés tout à faits satisfaisants ${ }^{10}$. Dès lors, si c'est bien cette logique technico-gestionnaire imposée, couplée de démocratisation imposée, inhérente au fantasme d'ingérence de l'ong qui explique l'échec d'Alizés Électrique, pourquoi cela n'a-t-il apparemment pas été le cas dans le cas d'Alizés Pompage? N'aurait-il pas été de bonne méthode scientifique d'aller tester cette hypothèse en allant enquêter dans des villages équipés d'éoliennes de pompage et analyser les modes de gestion réels, dix ans après? Cela aurait peut-être été l'occasion pour Sophie Caratini d'infirmer le fait qu'une part significative de ces éoliennes fonctionne toujours et de montrer que les mêmes contradictions internes aux comités sont la cause de l'arrêt, confirmant ainsi sa thèse. Mais cela aurait peut-être pu lui montrer comment les acteurs locaux ont su se réapproprier la gestion et la transformer, au-delà du modèle initial et de ses ambiguïtés éventuelles, pour en faire quelque chose de viable (quitte, peut-être à en « privatiser » la gestion comme au Niger [Oliver de Sardan \& Dagobi 2000]). Ou encore d'approfondir ce qui m'apparaît finalement comme l'apport principal de son enquête: "On ne saurait mettre sur le même plan les besoins en électricité et en eau potable. En pays maure, seule l'eau peut générer une dynamique véritablement "collective". [...] Personne ne saurait en être privé, car l'eau, c'est la vie, partant la survie du groupe, alors que l'électricité apparaît comme un luxe individuel » (ibid. : 81-82).

\section{Fantasme technico-gestionnaire ou différence de nature de service entre l'eau potable et l'électricité?}

Dans cette hypothèse, une des erreurs fondamentales du Gret ${ }^{11}$ aurait ainsi été de supposer que ce modèle de gestion, qu'il considérait avoir testé et validé dans le contexte social du Trarza, pouvait sans problèmes se transférer du pompage d'eau à l'électricité, sous-estimant ainsi les profondes différences entre ces deux types de service de base, en termes de complexité technique et économique, mais plus encore en termes de représentations locales et de volonté politique à assurer un service collectif. Ce qui questionne, effectivement, le choix d'un service accessible au plus grand nombre et de comités de gestion, pour un service de ce type, par rapport à un service privé, accessible à ceux qui ont les moyens de le payer. Si cela est juste, outre les recompositions institutionnelles au niveau national qu'il aurait été intéressant d'approfondir ${ }^{12}$, l'échec trouve d'abord sa source dans ce transfert hasardeux, dans le fait d'avoir considéré comme acquis, et n'ayant pas besoin de faire l'objet d'un travail d'analyse préalable et de débats approfondis avec les acteurs locaux, un élément qui ne l'était pas. 


\section{Peut-on refuser aux praticiens le bénéfice d'une posture compréhensive?}

21 Ces deux études ont pour point commun de centrer leur analyse sur les perceptions des acteurs locaux (ou en fait, plus exactement, sur un discours de chercheur censé s'appuyer sur ces perceptions), et de ne pas interroger l'intervention elle-même. Le cadre institutionnel et organisationnel ${ }^{13}$, les équipes et leur composition, les pratiques effectives des agents, l'analyse qu'ils font de la situation dans laquelle ils agissent et des problèmes posés par leur intervention : rien de tout cela ne fait partie de l'analyse.

C'est totalement absent chez Arditi, qui ne dit pas qui délivre le crédit, du BELACD ou de l'UCEC, et critique l'AFDI sur trois pages avec comme seul élément empirique « l'AFDI, qui est présente dans le sud du Tchad pour appuyer les actions en matière de traction animale» (Arditi 2004: 857). Sophie Caratini (2005: 74) est plus précise sur l'intervention elle-même, mais a pour parti pris affiché un principe d'asymétrie : sa démarche, telle qu'elle l'expose elle-même dans son article, repose sur un principe d'asymétrie, croisant les récits "des Mauritaniens » ${ }^{14}$ (mais en excluant les agents mauritaniens du projet) et littérature grise du projet. Elle a refusé de rencontrer et d'interroger les membres de l'ong qui ont participé à cette histoire (Mauritaniens compris) $)^{15}$.

Les deux chercheurs refusent ainsi le principe de symétrie dans le traitement des « développeurs » et des « développés » mis en avant - à défaut d'être toujours pratiqué - par l'anthropologie du développement, "par l'enchevêtrement des logiques sociales » (Olivier de Sardan 2001) centrée sur « l'interface » entre acteurs. Ils refusent aussi aux praticiens le bénéfice d'une approche compréhensive, qu'ils revendiquent à juste titre pour leurs sujets d'étude habituels. Il est assez aisé de démontrer les biais que cela induit : d'une part, une naturalisation de « points de vue locaux » dont on a du mal à penser qu'ils ne soient pas plus divers ${ }^{16}$ et, d'autre part, une lecture biaisée de l'histoire du projet et de la démarche de l'équipe. Lors de notre entretien, Sophie Caratini nous a expliqué combien le caractère technique des rapports d'activités l'avait choquée et convaincue de la force de cette logique «technico-gestionnaire». Sans doute. Mais comment peut-on, d'un point de vue socio-anthropologique, considérer que la littérature grise, qui renvoie à des objectifs pratiques dans le suivi du projet et la relation avec le bailleur et le maitre d'ouvrage, traduit suffisamment bien les points de vue et les pratiques pour qu'il n'y ait pas besoin d'interroger les acteurs de chair et d'os? Un tel choix permet certes à Sophie Caratini de défendre sa vision des développeurs, "ingénieurs qui s'attachent à fomenter des systèmes d'autocontrôle pour protéger leurs machines" (Caratini 2005: 75), enfermés dans cette logique « technico-gestionnaire » et leur " fantasme d'ingérence » (ibid. : 91), en lui évitant de la confronter dans l'enquête aux praticiens, acteurs de chair et d'os, qui sont certes français et ingénieurs pour une partie d'entre eux (ce qui induit incontestablement des positions sociales et des schémas de pensée), mais qui ne sont pas pour autant totalement prisonniers de leur culture d'ingénieur, et sans aucune compréhension des rapports sociaux locaux.

$\mathrm{Si}$, à défaut de pouvoir observer les pratiques, elle avait enquêté sur elles, elle aurait trouvé un choix initial - clair mais pas forcément très « atterri » dans le contexte local - en faveur d'un service accessible au plus grand nombre, une vision sans aucun doute 
trop fonctionnaliste des comités (mais partie intégrante de la "culture " développementiste de l'époque), une conception finalement assez floue de la démocratie. Elle aurait vu des dialogues avec les jemaa - effectifs mais en partie asymétriques et en partie aveugles sur les enjeux politiques locaux - pour essayer de définir en commun un dispositif viable; un souci plus ou moins explicite de coupler légitimité politique et critères minimum de capacités techniques (alphabétisation, être sur place) dans le choix des postes dans les comités de gestion nommés par la jemaa, etc. Le tout dans une vision "communautaire" du service de l'électricité, dont la fonctionnalité suppose que des règles soient définies et mises en œuvre. Cela explique des décalages, des impositions de logiques, des interférences maladroites, d'une ampleur qui serait à analyser. Et donc l'existence de conflits suscités ou aggravés par l'intervention, eux-aussi à qualifier et à analyser. Mais n'est-ce pas bien autre chose qu'une "intention de concrétiser un modèle d'organisation sociale porteur d'un fantasme d'ingérence on ne peut plus explicite » (ibid.)?

Loin d'être dans une démarche inductive, partant de l'analyse empirique fine d'un cas permettant de remonter à une interprétation plus générale, nos deux auteurs utilisent le projet qu'ils étudient comme "illustration" de leur thèse, sous couvert d'une enquête de terrain dont la durée est ambiguë ${ }^{17}$. Ils mêlent ainsi un populisme "idéologique " à une posture de "déconstructions du discours", qui "permet de se livrer sans risques à des énoncés catégoriques, assénés sans souci des nuances, et sans tenir le moindre compte de contre-exemples éventuels [...]» (Olivier de Sardan 2001 : 735). Dès lors que ces travaux prétendent analyser des interventions concrètes, mettent en avant des ONG précises, une telle démarche relevant alors des « délices douteux de la stigmatisation » (Le Naëlou \& Freyss $2004:$ 730), pose en plus de sérieux problèmes déontologiques.

\section{Les projets de développement comme construits sociotechniques}

26 Malgré le postulat initial de l'APAD (1991), qui voulait faire une anthropologie des " développeurs » au même titre qu'une anthropologie des "développés », le monde des développeurs n'a jusqu'à récemment pas vraiment fait l'objet de travaux empiriques approfondis de la part des anthropologues de langue française. Rares sont ceux qui, comme Berche (1998), Atlani-Duault (2005), Le Meur (2006), ont réellement pris les dispositifs d'intervention comme objets, ou se sont intéressés sérieusement aux pratiques et représentations des développeurs de chair et d'os impliqués dedans (de Noray 2000 ; Recondo 2006) ${ }^{18}$.

Cette absence laisse dans l'ombre des dimensions importantes de ce qu'est l'intervention de développement. Au-delà des raisonnements généraux sur les cadres cognitifs des développeurs (Olivier de Sardan 1995) et de la production et reproduction de l'ignorance dans le système d'aide (Jacob 2000), on ne sait finalement pas grandchose de la façon dont pensent les acteurs de chair et d'os, et dont ils perçoivent et gèrent les contradictions entre leurs schémas de pensée et les réalités qu'ils perçoivent sur le terrain. Au-delà du constat - finalement sociologiquement banal - que toute intervention suscite des enjeux et des conflits, on ne sait pas si, et dans quelle mesure, les dispositifs organisationnels et les cadres contractuels (avec l'État, avec les bailleurs) ont un impact sur les stratégies des projets et leurs pratiques, à travers le cadrage qu'ils 
imposent à l'action, des logiques de décaissement plus ou moins prégnantes, la possibilité ou non de réajuster l'action en cours de route, les mécanismes de redevabilité qu'ils intègrent ou non (et vis-à-vis de quels acteurs).

Considérant implicitement l'ensemble des acteurs côté "développeurs" comme un ensemble homogène, on ne dit rien des jeux d'acteurs complexes entre équipes projets, acteurs publics, bailleurs de fonds, pour légitimer une action, négocier un soutien ou des ajustements, et avec les acteurs locaux pour négocier le droit à agir (Mosse 2005), ni des évolutions dans le temps des dispositifs, des stratégies et des pratiques, en fonction de ces négociations, de la confrontation au réel des présupposés initiaux, des rapports de force entre acteurs.

C'est finalement une bonne part de ce qui fait la réalité pratique, d'une intervention de développement qui échappe à l'analyse. Tout projet peut en effet se lire comme un construit sociotechnique (Latour 1992), couplant dimensions techniques, organisationnelles, institutionnelles, sociales, politiques, etc. D'une conception sur le papier portée par quelques acteurs (elle-même issue de connaissances, d'expériences, de négociations variées, et donc dans un rapport variable avec la réalité), un projet doit pour se " réaliser » (pour devenir réalité) recruter et consolider un réseau de soutiens (depuis les conseils d'administrations des bailleurs jusqu'aux populations locales), renégocier ses postulats, faire face à des événements imprévus. Les acteurs qui le portent doivent mettre et faire fonctionner ensemble des éléments techniques (des machines, des éoliennes, des batteries, des lampes, ou des montants de crédit, des taux d'intérêt, des modalités de remboursement; des «non-humains » dirait Latour) et des acteurs de chair et d'os (des responsables politiques villageois, des habitants, des agents du ministère de l'Énergie, des agents du projet, des agents du bailleur, des consultants pour Alizés Électrique, des membres du BELACD, des responsables paysans d'UCEC, des emprunteurs et des emprunteuses, etc.). Chacun de ces acteurs a ses logiques, ses intérêts, qu'il faut prendre en compte. Les éléments techniques résistent ou non à s'emboîter, entre eux et avec les acteurs (les modalités de crédit sont pertinentes, ou pas ; les modes de gestion des éoliennes font sens ou non dans l'espace social local). Les équipes des projets consacrent (à plus ou moins bon escient) une part importante de leur action à essayer de faire marcher ensemble tout cela, à négocier les consentements, à modifier leurs pratiques pour prendre en compte les intérêts des autres acteurs, réussissant ou non à construire des dispositifs fonctionnels et à les stabiliser, dans un processus constant de négociation et d'ajustements des pratiques, des discours et du lien entre pratiques et discours. Analysant rétrospectivement l'expérience d'un projet de développement rural en Inde et sa propre contribution en tant que consultant anthropologue, Mosse (2005:5-8) s'appuie sur Latour pour montrer que le succès ou l'échec des projets «n'est pas donné dès le départ mais émerge de leur capacité en continu à recruter des appuis et ainsi à imposer [leur] cohérence croissante à ceux qui les mettent en question ou s'y opposent ${ }^{19}$. Mais cette «imposition de cohérence » n'est pas que du rapport de force, c'est aussi le fruit de ces ajustements et renégociations, de stratégies de mobilisation, d'intéressement et d'enrôlement des acteurs (Callon 1986). De fait, « un projet technique [ou de développement] n'est pas réaliste ou irréaliste, il se réalise ou se déréalise progressivement » (Latour 1992: 77) (au sens de " devenir réalité »). "Tout peut se réaliser ou se déréaliser en fonction des chaînes continues de traductions. Il faut toujours continuer à intéresser, à séduire, à traduire les intérêts » (ibid. : 78). 

réussite ou l'échec de projets de développement ne sont pas donnés a priori, même si les choix initiaux, les hypothèses de base, les cadres institutionnels constituent des sentiers de dépendance forts, et doivent donc être discutés. Les projets de développement sont le résultat du processus d'autant plus complexes qu'ils mettent en jeu des acteurs très hétérogènes dont les logiques sont peu lisibles pour les tiers; qu'ils agissent en situation de forte incertitude; que cette dimension processuelle est niée dans des « cadres logiques" inspirés de la gestion de projets industriels (Giovalucchi \& Olivier de Sardan 2009) et des contraintes bureaucratiques et financières fortes. L'asymétrie des moyens financiers permet aisément "d'acheter» la participation apparente des acteurs tiers (l'administration, les populations, etc.) à coup de corruption ou de per diem. Mais il existe aussi des interventions qui, dans ces configurations complexes, essaient de mettre l'accent sur la recherche de la pertinence de l'action pour les acteurs locaux, sur la construction progressive de «l'adéquation " de l'offre (Korten 1980), sur la construction institutionnelle (Barrau \& Frenoux 2010) et la stabilisation d'un soutien politique.

De la même façon que les recherches sur l'État tendent à donner à voir " un État sans fonctionnaires " (Copans 2001), les sciences sociales francophones - et l'anthropologie en particulier - montrent fréquemment un système d'aide sans développeurs. Cette occultation des dispositifs d'intervention et des développeurs a différentes causes : les ambiguïtés structurelles du "développement" et les nombreux effets pervers qu'engendre le système d'aide semblent suffire à expliquer les échecs et décalages; de nombreux projets fonctionnent sur une illusion et manipulent l'information (Arditi 2005) ; différentes recherches montrent que, localement, les populations ne distinguent guère entre les différents projets qui interviennent dans leur zone, et que les débats internes aux développeurs sur les stratégies et les dispositifs ont peu de traduction concrète pour eux. Par ailleurs, la force du discours développementiste et de sa rhétorique est telle que de nombreux chercheurs craignent de se laisser contaminer et veulent garder un cordon « sanitaire » avec les développeurs, ou se positionnent sur un registre critique de principe.

33 Cet état de fait pose cependant problème d'un point de vue de construction de l'objet et de méthode : dès lors que l'on prend des interventions de développement comme objets de recherche, peut-on faire l'impasse sur des analyses contextualisées, prenant acte de la diversité de configurations ? Peut-on refuser aux « développeurs » les bénéfices de ce qui est au fondement de la démarche anthropologique, et que l'on défend pour ses sujets d'études habituels, c'est-à-dire une démarche compréhensive, tentant de restituer les faits sociaux à partir de la façon dont les acteurs concernés (les acteurs des ONG au même titre que les populations locales) les vivent et les pensent ?

Au-delà d'interprétations discutables en termes scientifiques, une telle posture a également pour effet de laisser dans l'ombre des dimensions importantes de ce qu'est l'intervention de développement en pratique: les représentations et la capacité réflexive des praticiens, la façon dont se construit et se négocie l'action entre référentiels, cadres institutionnels, et jeux d'acteurs ; les processus de négociation et de médiation complexes, mobilisant un ensemble large d'acteurs (populations, représentants, élus, techniciens des services techniques ou des oNG, bailleurs, etc.) et qui déterminent l'histoire et la «réussite " ou «l'échec» d'un projet, à travers 
renégociations et compromis, glissements de stratégie, bifurcations, sentiers de dépendance, etc. Comme le souligne Mosse (2005:8), « La gouvernance apportée par les schémas de développement ne peut pas être imposée : elle réclame de la collaboration et des compromis ".

S'intéresser aux projets de développement comme processus sociotechniques, prendre les dispositifs d'intervention comme objet de recherche socio-anthropologique contribueraient de plus à décloisonner la recherche africaniste, à travers un objet susceptible de travaux comparatifs à l'échelle internationale : le projet comme mode d'action publique (Pinson 2004). Tout en étant inscrit dans des configurations politiques et institutionnelles spécifiques, liées aux rapports Nord/Sud, à l'histoire politique et institutionnelle des pays, au poids de l'aide, le projet de développement dans les pays africains n'est en effet qu'une modalité particulière de l'intervention sociale et du projet comme mode d'action publique, avec des démarches et des outils qui ne sont pas radicalement différents de ceux mis en œuvre au Nord, qui sont, eux, un objet légitime pour la sociologie politique. Cette mise en perspective comparative du projet comme mode d'action publique ne permettrait-elle pas de mieux distinguer, dans les analyses sur le développement, ce qui relève des dispositifs d'intervention, de leur culture, de leurs outils (et des contradictions qui leur sont propres) et ce qui relève des configurations sociales, politiques et institutionnelles (nationales comme internationales) au sein desquelles ces dispositifs s'inscrivent et qui les conditionnent fortement?

\section{BIBLIOGRAPHIE}

ARDITI, C. 2004 « Des paysans plus professionnels que les développeurs ? L'exemple du coton au Tchad (1930-2002) », Revue Tiers Monde, « ONG, les pièges de la professionnalisation », numéro spécial, XLV, $180: 841-864$.

- 2005 « Niger : chronique d'une évaluation censurée », Revue Tiers Monde, XLVI, 184 : 861-881.

ATLANI-DUAULT, L. 2005 Au bonheur des autres. Anthropologie de l'aide humanitaire, Nanterre, Société d'Ethnologie.

BARÉ, J.-F. (DIR.) 2006 Paroles d'experts. Études sur la pensée institutionnelle du développement, Paris, Karthala.

BARRAU, É. \& FRENOUX, C. 2010 «Vers l'institutionnalisation d'une délégation communautaire ? Le cas de l'accès à l'eau potable dans les quartiers défavorisés de Port-au-Prince », Revue Tiers Monde, $203: 123-140$.

BERCHE, T. 1998 Anthropologie et santé en pays dogon, Paris, Karthala.

BOUSSO, P. ET AL. 1997 L'impact micro-économique du crédit rural au Cambodge, Paris, Éditions du Gret (« Études et Travaux »).

CALLON, M. 1986 «Éléments pour une sociologie de la traduction. La domestication des coquilles Saint-Jacques et des marins dans la baie de Saint-Brieuc ", L'année sociologique, 36 :170-208. 
CARATINI, S. 2001 Projet ALER, volet « kits solaires », Note de synthèse, décembre, AFD.

- 2002 Projet ALER, volet « kits solaires », Rapport de synthèse, mars, AFD.

- 2005 « Le projet “Alizés Électrique" ou les paradoxes du rapport de développement », Autrepart,

"Les ONG à l'heure de la "bonne gouvernance” ", numéro spécial, 35 : 73-95.

COHEN, M. 2001 Connaître la clientèle des IMF : Outils d'analyse pour les praticiens de la microfinance, Paris, Document de travail de AIMS/USAID (version anglaise de 2000).

COLIN, J.-P., LE MEUR, P.-Y. \& LÉONARD, É. 2010 « Identifier les droits et dicter le droit. La politique des programmes de formalisation des droits fonciers ", in J.-P. COLIN, P.-Y. LE MEUR \& É. LÉONARD (dir.), Les politiques d'enregistrement des droits fonciers. Du cadre légal aux pratiques locales, Paris, Karthala : 5-67.

COPANS, J. 2001 « Afrique noire : un État sans fonctionnaires ? ", Autrepart, « Les fonctionnaires du Sud entre deux eaux », numéro spécial, 20 :11-26.

DAUVIN, P. \& SIMÉANT, J. 2002 Le travail humanitaire. Les acteurs des ONG. Du siège au terrain, Paris, Presses de Sciences Po.

FERGUSSON, J. 1990 The Anti-Politics Machine. "Development », Depoliticization, and Bureaucratic Power in Lesotho, Minneapolis, University Press of Minnesota.

GIOVALUCCHI, F. \& OLIVIER DE SARDAN, J.-P. 2009 « Planification, gestion et politique dans l'aide au développement : le cadre logique, outil et miroir des développeurs ", Revue Tiers Monde, 198 : 383-406.

HUCHON, X. 2000 Confrontations entre actions de microfinance et logiques paysannes, département de Mayo Dallol, Tchad, Mémoire de DESS, Nogent-sur-Marne, IEDES/ UCEC.

JACOB, J.-P. (DIR.) 2000 Sciences sociales et coopération en Afrique : les rendez-vous manqués, ParisGénève, PUF-IUED (« Nouveaux cahiers de l'IUED, 10 »).

JACOB, J.-P. 2010 « "Une brousse connue ne peut pas bouffer un fils de la terre !” Droits sur la terre et sociologie du développement dans le cadre d'une opération de sécurisation foncière (Ganzourgou, Burkina Faso) », in J.-P. COLIN, P.-Y. LE MEUR \& É. LÉONARD (dir.), op. cit. : 167-194.

KORTEN, D. 1980 « Community Organization and Rural Development : A Learning Process Approach ", Public Administration Review, septembre-octobre (version française partielle, L'intervention sociale comme processus d'apprentissage, Paris, Gret [« Coopérer Aujourd'hui, 48 »], 2006).

LATouR, B. 1992 Aramis ou l'amour des techniques, Paris, La Découverte.

LAVIGNE DELVILLE, P. 2007 Prendre au sérieux les pratiques des développeurs : une étape nécessaire de l'analyse critique des interventions des ONG ?, Paris, Gret ("Coopérer Aujourd'hui, 53 »).

LE MEUR, P.-Y. 2006 « Governing Land, Translating Rights : The Rural Land Plan in Benin », in D. LEWIS \& D. MOSSE (eds.), Development Brokers and Translators, Bloomfield, Kumarian Press : 75-100.

LE MEUR, P.-Y. \& LAVIGNE DELVILLE, P. 2009 « Le développement rural et la gouvernance des ressources ", in L. VIDAL \& L. ATLANI-DUAULT (dir.), Anthropologie de l'aide humanitaire et du développement, Paris, Armand Colin : 73-96.

LE NAËLOU, A. \& FREYSS, J. (DIR.) 2004 « ONG, les pièges de la professionnalisation », numéro spécial, Revue Tiers Monde, XLV, 180.

LI, T. M. 2007 The Will to Improve. Governmentality, Development, and the Practice of Politics, DurhamLondon, Duke University Press. 
MATTHIEUSSENT, S., CARLIER, R. \& LAVIGNE DELVILLE, P. 2005 Un projet d'électrification rurale en Mauritanie (1995-2000). Alizés Électrique : histoire et enjeux d'une tentative de construction d'un service durable, Paris, Gret («Études et travaux en ligne, $6 »)$.

MEYER, J.-B. 1997 Experts en mission. Les coulisses d'un transfert de technologie, Paris, Karthala.

MONGBO, R. 1995 The Appropriation and Dismembering of Development Intervention. Policy, Discourse and Practice in the Field of Rural Development in Benin, Ph. D. Thesis, Landbouw, Universiteit Wageningen.

MOSSE, D. 2005 Cultivating Development. An Ethnography of Aid Policy and Practice, London, Pluto

Press.

DE NORAY, M. L. 2000 « L'aide extérieure vue par les agents de développement maliens », Autrepart, $13: 109-125$.

OLIVIER DE SARDAN, J.-P. 1995 Anthropologie et développement, essai en socio-anthropologie du changement social, Paris, APAD-Karthala.

- 2001 «Les trois approches en anthropologie du développement », Revue Tiers Monde, XLII, 168 : 729-754.

OLIVIER DE SARDAN, J.-P. \& DAGOBI, A. E. 2000 « La gestion communautaire sert-elle l'intérêt public? Le cas de l'hydraulique villageoise au Niger », Politique africaine, 80 : 153-168.

PINSON, G. 2004 « Le projet urbain comme instrument d'action publique », in P. LASCOUMES \& P. LE GALÈs (dir.), Gouverner par les instruments, Paris, Presses de Sciences Po («Gouvernances ») :

199-233.

RECONDO, D. 2006 « Tailleurs de démocratie : l'assistance électorale de l'ONU racontée par ses artisans ", in J.-F. BARÉ (dir.), Paroles d'experts. Études sur la pensée institutionnelle du développement, Paris, Karthala : 29-56.

ROCHER, M. 2002 Travail de capitalisation. Alizés Électrique, rapport interne, Paris, Gret.

\section{NOTES}

1. Je mobilise sur ces deux cas, de façon condensée, une analyse développée dans LAVIGNE DELVILLE (2007).

2. Pour reprendre la distinction proposée par OLIVIER DE SARDAN (1995).

3. Je remercie Lætitia Atlani-Duault, Bernard Gay, Pierre-Yves Le Meur, et Jean-Pierre Olivier de Sardan pour leurs commentaires sur des versions successives de ce texte. Les analyses proposées ici n'engagent bien sûr que moi.

4. Pour des questions de place, je ne développe pas ici le cas de l'AFDI.

5. Voir BOUSSO ET AL. (1997 : 23-27), autour de la notion de fongilibité du crédit (quelque soit l'objet déclaré, l'argent passe de toutes façons par la trésorerie familiale). C'est à partir de 2000 (en anglais), 2001 (en français) que les outils méthodologiques intègrent l'analyse de l'utilisation du crédit partant du constat du décalage entre objet annoncé et usage réel (COHEN 2001).

6. On ne sait cependant pas s'il s'agit de discours publics ou privés, des individus ou de l'institution. Les rapports entre ces niveaux de discours auraient pu être intéressants à approfondir. 
7. Ce projet, mené par le Gret, dont j'ai dirigé la direction scientifique de 1999 à 2008, s'est déroulé avant que je prenne ce poste. Je n'y ai pas fait d'enquêtes de terrain mais ai encadré une analyse rétrospective de ce projet, menée sur dossier au siège du Gret (MATTHIEUSSENT ET AL. 2005) en m'appuyant sur des enquêtes de terrain réalisées en 2001.

8. Réalisées sur demande de l'AFD, qui souhaitait comprendre les blocages du projet ADER, conçu en partie à partir de l'expérience d'Alizés Électrique (CARATINI 2001, 2002).

9. Une partie non négligeable de ce qui ressort des récits rétrospectifs, potentiellement marqués par cette amertume, peut s'expliquer dans ce cadre d'interprétation, y compris le fait que cette déliquescence ait suscité ou aggravé les conflits et contradictions liés aux comités, mis des gérants en difficultés ou ait fourni à d'autres des opportunités de détournement, en tout état de cause ait été utilisée dans les conflits politiques locaux entre factions.

10. Le Gret n'a pas fait d'évaluation ex post de ce projet, mais d'après les informations qu'il détient, la majorité des éoliennes fonctionnaient, 10 ans après, ce qui est remarquable pour un projet de ce type, entre autres grâce à la mutuelle de maintenance.

11. Il y en a d'autres, en termes institutionnels et de démarche de recherche-action, et qui sont analysés dans MATTHIEUSSENT ET AL. (2005).

12. Quels enjeux du renforcement de la maîtrise d'ouvrage publique pour l'État mauritanien sur ce projet? Quelles stratégies de l'administration mauritanienne par rapport à l'imposition de la CELED ? Quelles logiques de nomination des responsables ?, etc.

13. Paradoxalement, alors que Caratini se focalise dans son article sur le seul Gret, sorte de "deus ex machina» dans les villages, ses rapports de mission pour l'AFD (CARATINI 2001, 2002) proposent un questionnement plus large (et me semble-t-il plus pertinent), autour des enjeux du projet entre État, AFD, Gret et IED (Innovation énergie développement, le bureau d'études qui a gagné l'appel d'offres d'appui à l'ADER). Elle y souligne entre autres que le durcissement des procédures est plus le fait de l'IED que du Gret...

14. Mes italiques.

15. Option affirmée lors d'un entretien à mon initiative (mars 2005).

16. Par exemple sur Alizés Électrique, entre autorités villageoises, factions rivales, membres de comités de gestion, anciens clients, anciens non-clients, agents du service de l'électricité, responsables des politiques de l'Énergie, etc.

17. Une fréquentation au cours de missions, la lecture de rapports, pour Arditi (comm. pers., Paris, 2006). Caratini (2005: 73) annonce une série de missions de terrain, mais les premières en tous cas étaient davantage consacrées au projet ADER. Ainsi la mission de 2002 a contenu trois jours de terrain pour cinq villages, dont deux concernés par le projet Alizés Électrique (CARATINI 2002 : 14).

18. BARÉ (2006) a tenté à juste titre d'ouvrir la question de « la pensée institutionnelle du développement ", mais peu d'articles de l'ouvrage sont en fait véritablement centrés sur cette question. On ajoutera MEYER (1997) en sociologie, et DAUVIN et SIMÉANT (2002) côté science politique, sur l'humanitaire et pour des publications récentes, voir JACOB (2010), COLIN ET AL. (2010). En anglais, mentionnons en particulier FERGUSSON (1990), MONGBO (1995), MOSSE (2005), LI (2007). Notons que Atlani-Duault, Mongbo, Mosse et 
Berche ont tous été impliqués dans les projets, dans ce qu'Olivier de Sardan appelle une situation de dédoublement statutaire, ce qui n'est sans doute pas sans lien avec la finesse de leurs analyses (LE MEUR \& LAVIGNE DELVILLE 2009).

19. Ma traduction.

\section{RÉSUMÉS}

Résumé

Lorsqu'ils analysent les projets de développement, certains anthropologues refusent d'étudier de façon symétrique les "développeurs" et les "développés", avec la même démarche compréhensive, wébérienne, qu'ils revendiquent pourtant légitimement pour les populations qu'ils étudient habituellement. À partir de deux cas, ce texte montre qu'une telle posture induit des problèmes méthodologiques et des interprétations discutables. Plus largement, l'anthropologie française du développement tend à négliger des parts importantes de ce qui constitue l'intervention de développement (les configurations institutionnelles, la réflexivité des agents de développement, les projets comme dispositifs sociotechniques, etc.) et dont la prise en compte éclaire de nouvelles facettes des projets de développement et des rapports entre « développeurs » et « développés ».

Abstract

When studying development projects, some anthropologists refuse to investigate symmetrically "developed" and "developers", with the same Weberian comprehensive postulate they claim for the populations they usually study. On the basis of two such cases, this paper shows that this leads to an inconsistent demonstration and a biased interpretation. While claiming such a symmetric methodology, numbers of studies in French anthropology of development tend to neglect the institutional frameworks of development projects and developers' concrete practices and representations. Important parts of what are concretely development projects, such as institutional configurations, practitioners' reflexivity, projects as sociotechnique processes, are thus overlooked in these studies, even if they shed new light on the reality of such projects.

\section{INDEX}

Keywords : Symmetric Analysis, Anthropology, Methodology, NGOs, Development Projects

Mots-clés : analyse symétrique, anthropologie, méthodologie, ONG, projets de développement

\section{AUTEUR}

PHILIPPE LAVIGNE DELVILLE

GRET/LASDEL, en accueil à l'IRD (UR 201 Développement et sociétés) et affecté au LASDEL (Niger). 